\title{
E-Learning Platform Development using A Model Driven Methodology Approach
}

\author{
P. Subba Rao ${ }^{1}$, K.B.S. Sastry ${ }^{2}$ and Hye-jin Kim ${ }^{3 *}$ \\ ${ }^{1}$ Dept. of Information Technology, VFSTR University \\ Vadlamudi, Guntur, India \\ ${ }^{2}$ Dept. of Computer Science, Andhra Loyola College \\ Vijayawada, India \\ ${ }^{3}$ Dept. of Child Welfare, Vision University College of Jeonju
}

235, Cheonjam-ro, Wansan-gu, Jeonju-city, Cheonbuk-do 560-760, South Korea 1subbarao.parem@gmail.com, ${ }^{2}$ sastrykbs@gmail.com, ${ }^{3}$ hyejinaa@daum.net

\begin{abstract}
These days, new advances and stages are developing and evolking continually, which suggests a high exertion for creating of complex frameworks for example, E-learning stage. This circumstance produces distinctive issues identified with compactness, reusability, versatility, incorporation and interoperability. The Object Management Group (OMG) proposes the Model Driven Architecture (MDA), which enhances mobility of uses by permiting the same model to be acknowledged on various stages Swch MDA characterizes a structural engineering focused around platform independem models (PIM) and platform specific models (PSM). The part approach means to outline and create frameworks from pre-assembled segments predesigned and pretested, to be reused in different applications, which would encourage application's support and development, would advance flexibility and configurability with a specific end goal to create new peoullarities. In this work we introduce LMSGENERATOR, a multi-target Learning administration framework generator with a model-driven procedure focused around MDA methodology coupled with part approach. Taking into account generative programming, from client determinations (abstract models) and the fancied advances, programming blocks will be created and amassed to deliver a complete arrangement adjusted to zone and clients' necessities. Thi paper concentrates on the change guidelines actualized in the LMSGENERATOR centers .Also; it introduces a careful investigation to delineate this proposal.
\end{abstract}

Keywords: E-learning, learning management system (LMS), model driven ensineering (MDE), model driven Architecture (MDA), transformation approach, $X M L$ technologies

\section{Introduction}

The significant downside of data frameworks is their expanding many-sided quality and quick adaptability. Managing this circumstance, scientists and commercial ventures have conceded to the way that the arrangement of this issue

* Corresponding Author 
ought to bring about an ascent of models, and a clearer partition in the middle of business and innovation. This choice has served to pass the models from their pondering stage, which was lessened to the representation and documentation of machine frameworks, to a more gainful stage which conceives the utilization of models in the heart of the frameworks improvement cycle. That is the reason the Model Driven Engineering (MDE) has been conception. The Model Driven Engineering (MDE) is a late train of programming building that advances models in five star elements in programming improvement [1]. It is the subject of extraordinary enthusiasm from the scholastic exploration groups (IDM-Action [2]) and mechanical research facilities (Compuware [3], Softeam [4], Andromda [5], Xactium [6], and so forth...). It is a manifestation of generative building, by which all or piece of a machine application is created from formats [7]. In this new point of view, models involve an unmistakable spot among the ancient rarities of frameworks improvement and in return must be sufficiently exact and rich so they can be deciphered or changed by machines. The methodology of framework advancement can then be seen as an issue of changes [7] of somewhat requested models, every change taking one or more models as data and creating one or more models as yield, until executable relics. On the other hand, the outline and programming of complex applications, for example, E-leaning stage, Faced with the developing and changing needs of the zone, needs to utilize the benchmarks, and needs to receive the new practice of programmin $g$ designing: Model driven Engineering, Generative Programming and Component Engineering.

This article presents the rudiments of another methodology [8] to program virtual learning situations. In light of generative programming, from client details (unique models) and the wanted advances, programming blocks will be created and afterward amassed to deliver a complete arrangement adjusted to territory and clients' requirements. This thought is embedded at diverse levels in the outline and at the present time Learning administration framework generator called LMSGENERATOR. This work demonstrates how the model driven engineering (MDE) specifically the OMT vision of MDE (MDA) and the Generative programming can be consolidated to execute this System.

\section{MDA}

Persuaded that the model has turned into the real standard by which the software industry can lift the latch automation improvement, the OMG (Object Managemeht Group) discharged in 2000 his vision of MDE: MDA (Model-Driven Architecture) [9]. This is both a proposed construction modeling and an improvement approach.

The essential thought of MDA is to partition the practical particular of a framework from points of interest of its usage on a particular stage. Therefore, MDA characterizes a structural planning particular organized in a few sorts of models [10]: Computational Independent Model (CIM), Platform Independent Model (PIM), and Platform Specific Model (PSM), created from the PIM focused around the PDM (Platform Description Model). The CIM models are the necessities of a framework, its motivation is to aid in understanding the issue and to create a typical vocabulary for an area. In UML, the utilization case chart is a decent possibility to speak to a CIM. The PIM, otherwise called the investigation and configuration model, is a conceptual model free from any running stage. 


\section{LMSGENERATOR: A MODEL-DRIVEN METHODOLOGY}

\subsection{Architecture and Functionality}

LMSGENERATOR is the programmed execution of new learning administration framework programming methodology. In view of new improvement methodology of programming building, it permits, from a plan of action storehouse, to create business parts, and after that returns by amassing these segments to produce E-learning stages. It proposes a multi-programming methodology [8] that unions the model-driven designing, generative building and segment building. This accumulation of strategies and procedures makes our approach more suitable to the developing needs of the area and engineering towards more established and general methodologies.

LMSGENERATOR is [11] is an environment utilized for incorporation of business segments and era of different parts from model. It points the era of E-learning, executable on different runtime situations and speaking to criteria of versatility to distinctive client classes. In view of multi-layer and interconnected programming parts (Fig. 1), this generator gives these clients the capacity to:

- Maintains a repository of conceptual components (business model repository) (PIM) durable, customizable, flexible and reusable as wely as an extensible repository of platform execution model (PDM).

- Applies transformation, generation and refining nules into models (element of business model repository) in order to generate a business components runtime on the target platforms, using PDM.

- Integrates existing components in its business components repository.

- Describes and maintains models (stracture) of E-learning platform and stores them in a descriptive repository for use as needed.

- Generates distancedearning environments based on predefined models in the platform descriptive repository or by generating directly a new platform with the ability to save its structure in the dedicated repository.

- Deploys the environments in target execution platforms (on specific application seryers and database servers).

The following figure raentifies the software components that support the two generation phases of LMSGENERATOR which will be detailed in the following section, in particularMDA generation phase.

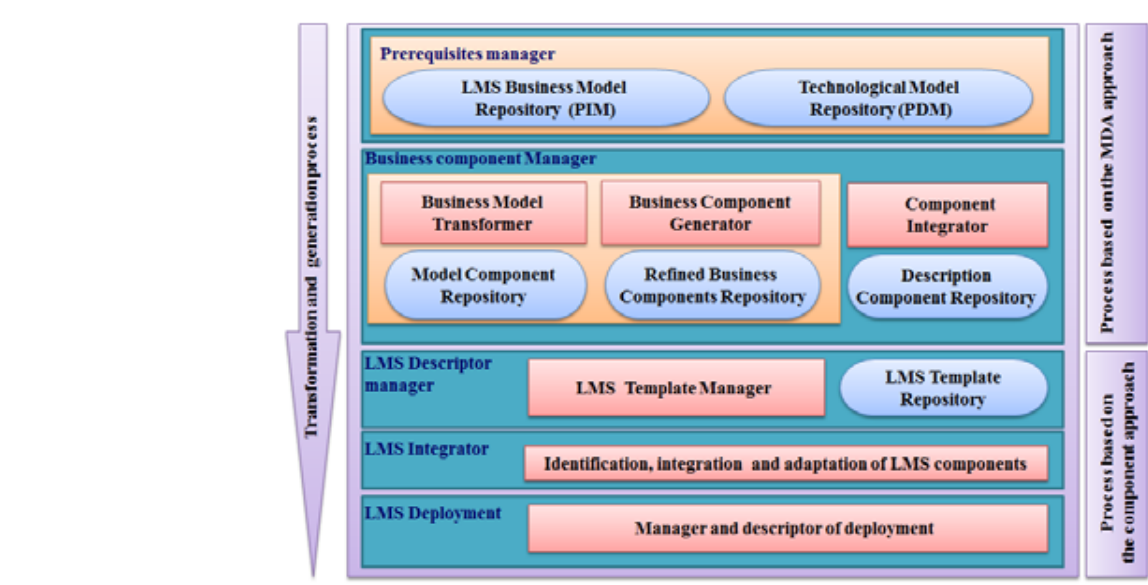

Figure 1. LMSGENERATOR Software Architecture 


\subsection{Generating Process Based on MDA}

This stage expects to assemble LMS business segments speaking to criteria of flexibility and subject to a target engineering (target runtime). The beginning stage of this stage is the components of our business vault. This store is made bankrupt model intended to be utilized as a part of the web. Hence we selected models that must appreciation the MVC ideal (Model-View-Controller) [13]. This programming pattern proposes the partition of the application into three sections:

- The model, which contains the logic and the state of the application;

- The view, which represents the user interface;

- The controller, which handles the synchronization between view and model.

Therefore our business model consists of three sub models:

- The business logic model to describe the business logic component

- The application logic model to structure and synchronize between the business and the presentation of the component.

- The presentation model to define the user interface, tegardles of the display means.

When the model is introduced, it experiences an era methodology focused around MDA methodology backed by two product parts of LMSGENERATOR: Business Model Transformer and Business Component Generator. Beginning from an applied business model(element of Business Model Repository), we can apply a set of change, deductions gaidelines and progressive enhancements to deliver a refined LMS business componentelement of Refined Business Component Repository), prepared to be utilized as an issue building square in the development of our learning stage. This erd stage comprises of two fundamental steps. The principal step intends to produce from a plan of action a segment display autonomously of the engineering communicated in XML document configuration, while the second uses the XML record to create a business part relying upon a arget innovation.

The initally step is focosed around an era methodology focused around model changes receinng the change by program methodology utilizing APIs controlling UML models gave being developed situations. Therefore, with negligible exertion, a segment model free from innovation can be immediately acquired by an arrangement of changes, mergers or refinements of the three sub models specified at one time (Fig. 2). This change by programming methodology is the charge of Busines SModel transformer (Fig. 1). This segment model, as close as could be expected-ander the circumstances to the needs of the application, indicating the administrations gave and obliged, enter, in its turn, in an era methodology upheld by Business Component

Generator (Fig. 1), receiving the change by format approach. This second step is focused around a situated of calculated models for innovation advancement characterized in our innovative model store (Fig. 1). 


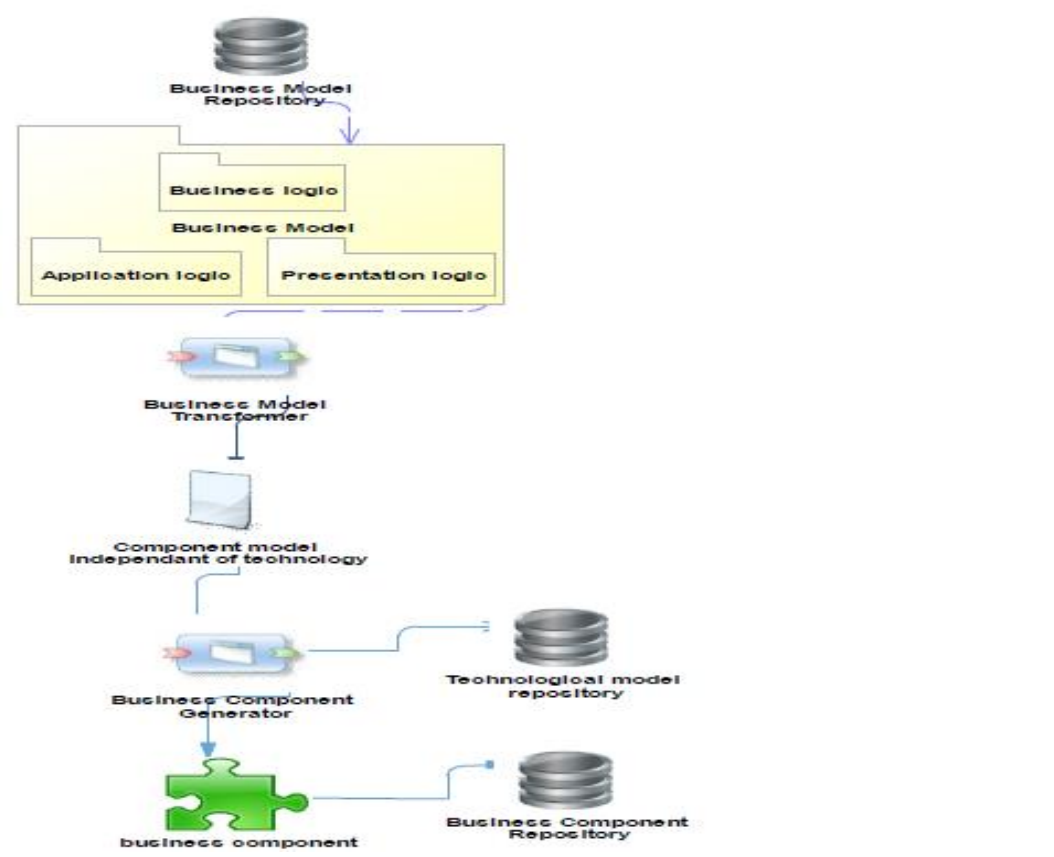

Figure 2. Generation Process based on MDA approach of LMSGENERATOR

\section{Case Study}

\subsection{The Model Specification}

In this area we have picked as plan of action, an allotment of the correspondence model introduced in 512$]$ that would serves as the discussion model. This non-concurrent correspondence mean is considered as an issue alone model and plays in the meantime a.critical part in E-learning stage. In the wake of transforming, we will be given a reusable business part that can be effectively coordinated into any stage. The principle prerequisite of e-learning stages, which is a piece of collective apparatuses, is the talk discussion.

The sarne instrument is ytilized as a part of web applications focused around collective work. Discourse gatherings - or news bunches - can make topical trades places where everyoody can communicate. They can be united or not by a mediator who is in charge of "separating" the inquiries or the answers and additionally oversees subjects.

\subsection{The Modeling Process}

Since the plan of action is the heart of our era process [16]. Its representation is a critical errand. For this we will take after the best suited methodology to create an element web application exhibited in [17]. From the get go, the needs will be displayed utilizing the UML utilization case (Figure 3). They will be spoken to by a more solid HMI model (Human Machine Interface) intended to respond to future clients. For our situation we utilized just make and erase operations to abstain from entangling our detailed analysis. 


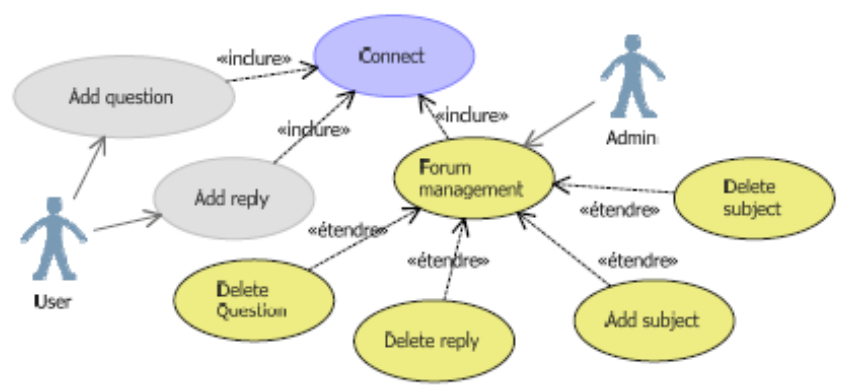

Figure 3. Use Case Diagram of Forum Discussion Model

In the connection of item arranged frameworks, the structure of the code is characterized by the product classes and their groupings into gatherings called bundles. So we need charts speaking to programming classes and demonstrating the information contained in them (called attributes), the administrations gave by those classes (called operations) including the connections between the operations. UML proposes the class graphs to pass on this data.

To present our research endeavor model, we will utilize a docupientation that has been proposed by I. Jacobson [18] and afterward promoted by RUP [19]. The Analysis classes they backer are partitioned into three classifications: Dialogs classes, controls classes, and the substance classes The Classes that permit cooperation between the site and its clients are called "dialogs".

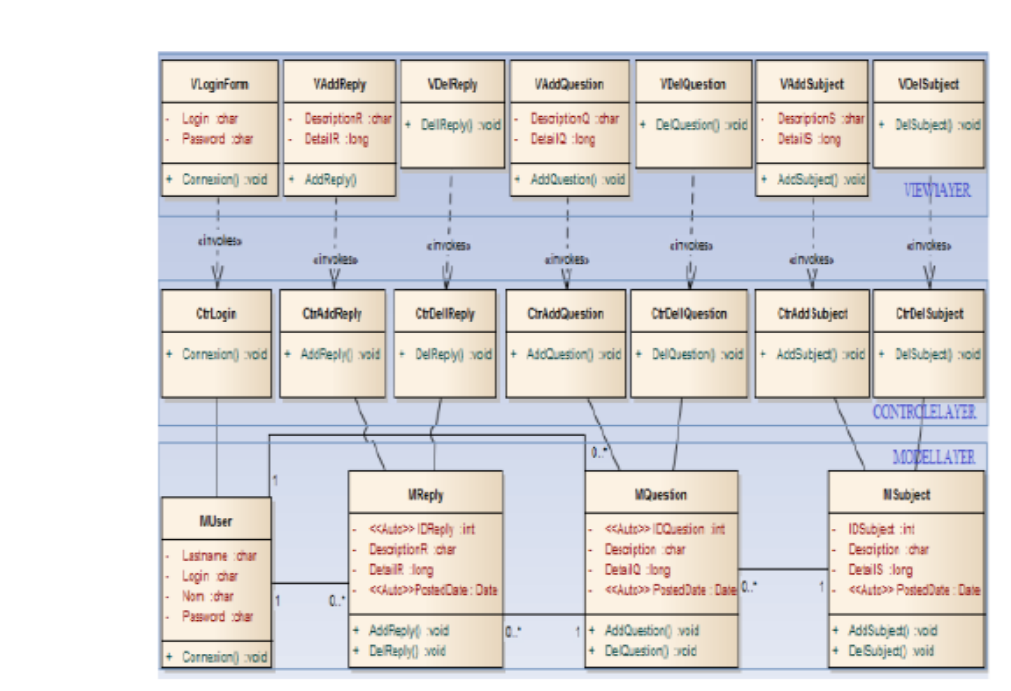

Figure 4. Class Diagram of Forum Discussion Model

\section{4.. PU to PIM Transformation}

To change over the model in Figure 4 in a XML document, we will utilize the programming change approach. Utilizing the API controlling of UML graphs, particularly those controlling class charts, we will create a document depicting our plan of action in a XML record configuration, partitioned into four segment in which we regard a meta-model change focus on that we have created and whose review is given in figure (Fig. 5).

The root component of our XML record is the component "Businesscomponent", this complex component has one quality "Name" and four sub-components: Viewlayer, Controlelayer, and Modellayerdaolayer. Viewlayer is 
the area speaking to the perspective share of our part. It incorporates all dialog classes changed into presentation pages "Viewpage" paying little mind to the engineering. These pages contain info fields (Input), showcase fields or hyperlinks (Join). The thing "Controlelayer" incorporates control classes changed into control pages "Conrolepage.

The calculation utilized as a part of this change is extremely basic: we crosses the class chart of our model class by class, if the class name starts with a "V", its portrayal will be included Viewlayer segment as Viewpage.

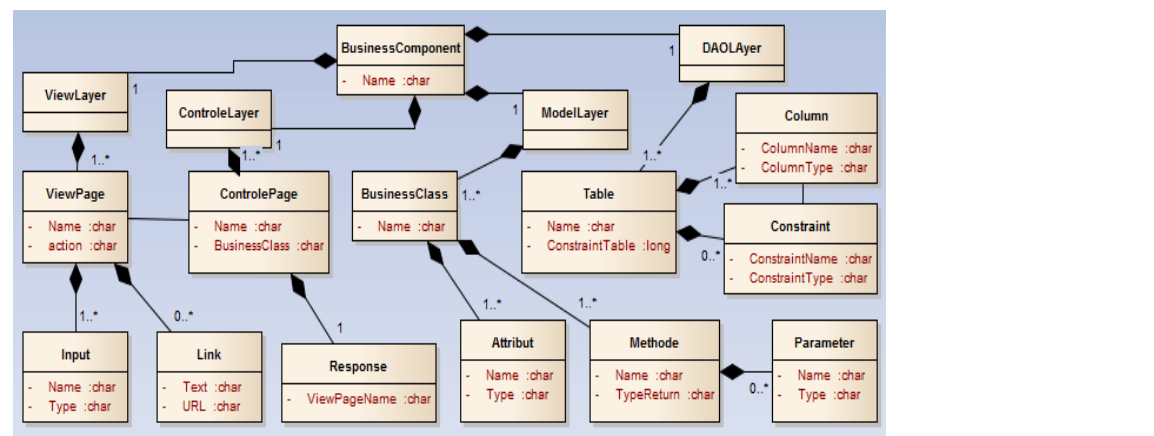

Figure 5. Meta-model Transformation Target

When you click on the catch, the structure is Cent to the control class determined in the activity property of "Viewpage". When the structure is recuperated by the control class, it istransformed urtilizing the system indicated as a part of "Methodecontrole" (Connection) of the class pointed out in "Businessclass" (Muser). At that point a reaction is planned as indicated by the consequence of technique execution. In tho practical necessity we can relate a table of the same portrayal as the business class "Muser". At this table we can include stipulations at the lable or section level.

\subsection{PIM to PSM Transformation}

We utilze XML document produced as a part of the past segment to create a business part reliant on a target engineering. We picked the J2ee especially MVC standard to settle pur research endeavor. This step is focused around generative building in which aPIM (XML record) is changed into a PSM. This change is focused around the format approach. From the details contained in the XML record we create completed antiquities. This change is focused around predefined innovative formats put away in our mechanical model archive. Here is the incomplete aftereffect of this change.

The main component of our era procedure is the application segment. It is an element web application regarding the Mvc2 ideal model. It comprises of three bundles: Viewlayer, Controllayer, and Modellayer. The Viewlayer bundle is the presentation layer of our application. It contains the accompanying JSP pages: Vaddquestion.jsp, Vaddreply.jsp, Vaddsubject.jsp, Vdelquestion.jsp, Vdelreply.jsp, Vdelsubject.jsp, and Vloginform.jsp Verrorlogin.jsp.

The second component of our era methodology is the script to make database produced under SQL group with the information portrayal dialect. The item social mapping is carried out physically by means of devices, for example, Hibernate right now being chipped away at to make this methodology programmed. 


\section{Conclusions}

Our methodology encourages the configuration of e-learning stages, through their development by gathering parts created from plan of action. It additionally quickens their advancement and arrangement by the guideline of programming reuse, as it encourages their improvement by giving an acceptable partition in the middle of defining and executing segments. The fundamental preference of our methodology is the attention of future advancements in innovation or space. These improvements are backed by adjusting (or making) generators connected with the models that will naturally engender changes over all situations and segments items.

We exhibited in this work, an outline of our methodology and its execution (LMSGENERATOR) and we concentrate on the era stage focused around MDA approach. A target meta-model was introduced to encourage understanding the change principles of PIM to PIM sorts. Future work would incorporate the conclusion of this generator, by characterizing era standards executed in these centers that are connected with PHP and .NET advances and by bolstering the innovative vault at present containing as of not longago just J2ee nodels utilized as a part of our careful investigation.

\section{References}

[1] J. Bézivin, "Sur les principes de base de 1 Ningénierie des modèles," RTSI-L'OBJET, vol. 10, no. 4, (2004), pp. 145-157.

[2] Action IDM. [Online]. Available: http:/www.actionidm.org

[3] OptimalJ - Model-driven developnent for Java. Electronic Source: Compuware. [Online]. Available: http://www.compuware.com/products/optimalj/, 2003.

[4] Softeam, Support de formation Objecteering 6/MDA, Modeler version 2.0, Janvier, (2008).

[5] AndroMDA. (2008). [Online]. Available: hrtp.//www.andromda.org/.

[6] XMF-Mosaic. Electroni Source. [Online] Available: http://www.xactium.com.

[7] S. Diaw, R. Lbath and B. Coulette. State of the art of software development based on model transformations [Online]. Available hitp://www.tsi.revuesonline.com/article.jsp?articleId=14833

[8] R. Dehbi, M. Talea, and A.Tragha "The generation approach of Multi-target learning management system," presented at the International Conference on Education Technology and Computer, Cape Town, South Arrica, (2012) Pugust 18-19.

[9] Soley, MDA (Model-Driven Architecture), White Paper, Draft 3.2, OMG Staff Strategy Group, vol. 27, (2000) November.

[10] X. Blanc, MDA er action : Ingénierie logicielle guidée par les modèles, Eyrolles, (2005).

[11] R. Dehbi, M. Tarea and A. Tragha, "Lmsgenerator: multi-target learning management system generator based on Generative Programming and Component Engineering," presented at the IEEE International Conference on Education and E-Learning Innovations, Sousse, Tunisia, (2012) July 1-3.

[12] R. Dehbi, M. Talea, and A. Tragha, "The modeling elements of LMSGENERATOR business repository," presented at the IEEE international Colloquium in Information Science and Technology, Fes, Morocco, (2012) October 22-24.

J. Galloway, Professional ASP.NET MVC 3, John Wiley \& Sons, 2011. [14] J. Pauli and G. Ponçon, Zend framework, Eyrolles, (2008).

[14] D. Alur, J. Crupi, and D. Malks, Core J2EE Patterns: Best Practices and Design Strategies, Prentice Hall, (2003).

[15] E. Evan, Domain-Driven Design: tackling complexity in the heart of software, Addison-wesley, (2003).

[16] P. Roques, UML2: Modéliser une application web, 4 ed. Eyrolles, vol. 1, (2007), pp. 14-21.

[17] I. Jacobson, G. Booch, and J. Rumbaugh, The Unified Software Development Process, 1 ed, Addison-Wesley, (1999) February 14.

[18] Best Practices for Software Development Teams, Rational Software White Paper, Rational Unified Process, TP026B, Rev 11/01. 